\title{
AN EFFICIENT ALGORITHM FOR THE BOTTLENECK PRODUCT RATE VARIATION PROBLEM WITH PRECEDENCE CONSTRAINTS
}

\author{
T. N. Dhamala \\ Central Department of Mathematics, Institute of Science and Technology \\ Tribhuvan University, P.O.Box. 13143, Kathmandu, Nepal \\ Corresponding address: dhamala@yahoo.com \\ Received 17 November, 2010; Revised 10 September, 2011
}

\begin{abstract}
We consider the problem of obtaining an optimal mixed-model sequence under the just-in-time environment. Industrial applications include the production planning, real-time scheduling, response time variability and networking. The single-level problems are already solved, but they are strongly NP-hard in the multi-levels. Here, we study a bottleneck product rate variation problem with a general objective where a given set of sequences serves as chain constraints. We extend the previous result of a similar problem with min-max deviation objective in single- level. We present a pseudo-polynomial algorithm that obtains an optimal solution for the considered objective. The results are valid for precedence constraints.
\end{abstract}

Keywords: integer programming, just-in-time sequencing, mixed-model systems, bottleneck product rate variation, precedence constraints.

\section{INTRODUCTION}

The main objective of the mixed-model just-in-time systems is to increase profit by reducing cost of diversified small-lot instead of large-lot producing only required parts in necessary quantity when needed. The flexible transfer lines have been implemented, where negligible switch over costs from one model to another have feasibility. This system satisfies the customer demands for a variety of products without incurring large shortages or holding large inventories. It has to utilize the elegant mathematical concept of equally penalizing jobs both for being tardy and for being early. One of the most important minimization problems is to determine a sequence such that it maintains the actual production level and the desired one as close to each other as possible during the production process. This minimizes the deviation between the actual and the ideal (desired) one and maintains the assembly line keeping rate of parts usage as constant as possible. Such sequences have been called balanced, fair or level. A schedule is the corresponding time table.

The single-level problems and the multi-level problems with pegging assumption are al- ready solved, but the multi-level problems are strongly NP-hard. If outputs at production levels which feed the final assembly level are dedicated to the final product into which they will be assembled, then the problem with pegging is equivalent to a weighted single-level problem which can then be minimized by modified algorithm for un-weighted single-level problem. The minimization of 
maximum deviation is co-NP, but the complexity of these problems remains open for the binary encoding [2].

Researchers and professionals have been considering different objective functions, like maximum or the sum deviations. There is no absolute understanding that the specific objective function among others is the best one. In this work, we sketch the past results with the classical bottleneck objective functions and give a modification of the existing algorithms, which fit, to our proposed more general maximum deviation objective. Hence, we generalize the previous results.

Research in mixed-model just-in-time sequencing problem begins after Monden [18] (see also [14]). Miltenburg [17] gives a nonlinear integer programming formulation of the single-level problem. Steiner and Yeomans [20] (see also [2]) solve the single-level problem for absolute deviation which is also applicable for multilevel with pegging assumption [19]. Steiner and Yeomans [19] prove that the cyclic sequences for maximum deviation are optimal. These results help on reducing the time complexity. Kubiak [16] gives a geometric proof and Brauner et al. [3] exploit the elegant concept of balanced words and gives an algebraic proof of the small deviation conjecture of [2]. Kovalyov et al. [15] illustrate the computational results. Corominas and Moreno [4] establishes optimality relations between different objective functions. For the recent survey and the accurate references, we refer to $[1,7,9]$.

Dhamala et al. [6] answer the question of [9] on the bound of squared-deviation and minimize this objective function. Recently, these results are generalized in [13]. Dhamala [8] and Dhamala and Kubiak [10] have added the first-order first-serve concept in studying the mixed-model just-in-time sequencing problem. Given a set of non-overlapping sequences as chain constraints, they give a pseudo-polynomial algorithm, which obtains an optimal solution to the whole instance that preserves the customers orders. The objective function they have considered is the absolute-deviation. This algorithm is also of pseudopolynomial time.

Very recently, Dhamala [5] extends the results and proposes a pseudopolynomial time algorithm by combining the above both concepts in $[8,6]$ to solve the bottleneck product rate variation problem with chain constraints and a squared deviation objective. In this paper, we give new extensions of the results in [5] for a more general objective, recently proposed by [13] and the precedence constraints.

The plan of the paper is as follows. Section 2 gives a brief overview of the mixedmodel just-in-time sequencing algorithms with different min-max objectives. In this section, we modify the formulation with additional chain constraints. Section 3 reviews the literature. In Section 4, we present a pseudo-polynoimal algorithm for our problem which is our main result. The final section concludes the paper. 


\section{OPTIMIZATION PROBLEMS}

For $i=1,2, \ldots, n$, given $n$ products (models) $i, n$ positive integers (demands) di and $n$ convex-symmetric functions $f i$ of deviation, all assuming minimum 0 at 0 , we consider the following nonlinear integer programming problem given in $[17,20]$. Find a sequence $s=s_{1} s_{2} \ldots s_{D}$ with total demand $D=\sum_{i}^{n} d_{i}$ where product $i$ occurs exactly $d i$ times that minimizes the following objective function. 1

$$
F(s)=\max _{i, k} f_{i}\left(x_{i k}-r_{i} k\right)
$$

where $x_{i k}$ represents the number of product $i$ copies in the prefix $s_{1} s_{2} \ldots s_{k}, k=1,2, \ldots, D$, and $r_{i}=\frac{d_{i}}{D}, i=1,2, \ldots, n$. To the function $\mathrm{F}$, there have been studied two objectives

$$
f_{i}\left(x_{i k}-r_{i} k\right)=\left\{\begin{array}{c}
\left(x_{i k}-r_{i} k\right)^{2} \\
\left|x_{i k}-r_{i} k\right|
\end{array}\right.
$$

of squared deviation and absolute deviation, respectively.

Its solution always keeps the actual production level xik and the desired production level ri $k$ as close to each other as possible all the times. The maximum deviation mixed-model just-in-time sequencing problem is also denoted by min-max problem.

The nonlinear integer programming problem modeled above should satisfy the following sets of the cardinality, monotonicity and integrality constraints, (see $[17,20])$ :

$$
\begin{array}{lc}
\sum_{i=1}^{n} x_{i, k}=k & k=1, \ldots, D \\
x_{i, D}=d_{i} & i=1, \ldots, n \\
x_{i, k} \leq x_{i, k+1} & \forall i \text { and } k=1, \ldots, D-1 \\
x_{i, k} \text { integer } \geq \mathbf{0} & i=1, \ldots, n, k=1, \ldots, D,
\end{array}
$$

The first constraint ensures that exactly $k$ units are scheduled in periods $\mathbf{1}$ through $k$, whereas the second constraint ensures that production requirements are met for each product. The third constraint guarantees that the total production of every product over $k$ is non-decreasing function, whereas the last one is integrality constraint. These four constraints jointly indicate that exactly 
one product is produced during each stage.

Note that the above formulation gives the following number-theoretic interpretation of JIT sequencing problem: given $n$ rational numbers $r i$, $i=1,2, \ldots, n$, with common denominator $D$, the problem is to find $n D$ integers xik which optimally approximate the sequence (kri) under the cardinality and monotonicity restrictions defined above (see [2], for the references).

The set of all feasible solutions satisfying the above constraints is denoted by the space $X=\left\{X \mid X=\left(x_{\downarrow} i k\right)_{\downarrow}(n \times D)\right\}$. Thus, the mixed-model just-in-time sequencing problem is equivalent to the following optimization problem

$$
\min \{F(s) \mid X \in X\}
$$

A feasible solution $s=s_{1} s_{2} \ldots s_{D}$ of the min-max problem of $\mathrm{n}$ models is called Bfeasible if maxi, $k$ fi (xik $\mathbf{I}$ ri $k) \leq B$ holds for the $n \times D$ matrix variables $X=(x i k)$. The restricted space of all B-feasible solutions is denoted by $x_{B}$.

We recall the model of [8] by adopting the constraints given by the following.

$$
\begin{aligned}
& \text { Chain }_{1}: u\left(n_{1}, D_{1}\right)=u\left(n_{1}, D_{1}\right)_{1} u\left(n_{1}, D_{1}\right)_{2} \ldots u\left(n_{1}, D_{1}\right) D_{1} \\
& \text { Chain }_{2}: u\left(n_{2}, D_{2}\right)=u\left(n_{2}, D_{2}\right)_{1} u\left(n_{2}, D_{2}\right)_{2} \ldots u\left(n_{2}, D_{2}\right) D_{2} \\
& \vdots \\
& \text { Chaint }: u\left(n_{\boldsymbol{t}}, D_{\boldsymbol{t}}\right)=u\left(n_{\boldsymbol{t}}, D_{\boldsymbol{t}}\right)_{1} u\left(n_{\boldsymbol{t}}, D_{\boldsymbol{t}}\right)_{2} \ldots u\left(n_{\boldsymbol{t}}, D_{\boldsymbol{t}}\right) D_{\boldsymbol{t}} \\
& \vdots
\end{aligned}
$$

Chainm $: u\left(n_{m}, D_{m}\right)=u\left(n_{m}, D_{m}\right)_{1} u\left(n_{m}, D_{m}\right)_{2} \quad \ldots u\left(n_{m}, D_{m}\right) D_{m}$

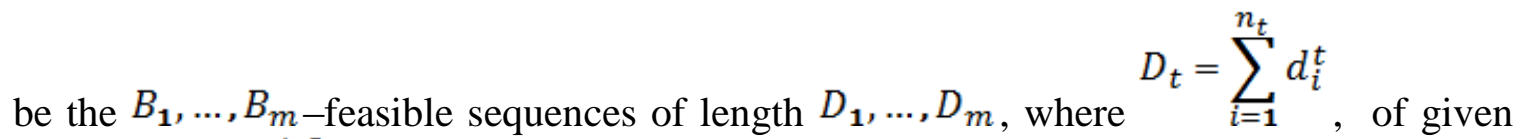
models $n_{t}, t=1,2, \ldots, m$, respectively. The system is called overlapping if there exists a common product in more than one chain. Here, we give algorithms for the problem with non overlapping system.

We extend the previous results and obtain a $B$-feasible sequence $\bar{s}=s_{1} s_{2} \ldots s_{D}$ with total demand $D=\sum_{t=1}^{m} D_{t}$ for mixed-model just-in-time sequencing problem such that the mapping satisfies $\bar{s}_{\left.\left(\mathbf{n}_{\mathbf{t}}, \mathbf{D}_{\mathbf{t}}\right)\right)}: \bar{s} \rightarrow u\left(\mathbf{n}_{\mathbf{t}}, \mathbf{D}_{\mathbf{t}}\right)$ for all $t=1,2, \ldots, m$ and has the least maximum deviation with a general objective, that is, $F(s) \geq F(s)$ for any sequence 
$s=s_{1} s_{2} \ldots s_{D}$ satisfying $s_{\left(\sum \mid u\left(n_{t}, D_{t}\right)\right)}: s \rightarrow u\left(n_{t}, D_{t}\right)$. The restriction mapping denoted as $\left.s_{(E} \mid u\left(n_{t}, D_{t}\right)\right)$ of the supper sequence $s$ to any given subsequence $u\left(n_{t}, D_{t}\right), t=1,2, \ldots, m$, yields the given sequence $u\left(n_{t}, D_{t}\right)$. Therefore, the supper sequence $s$ that contains $u\left(n_{t}, D_{t}\right)$ as its subsequence is order preserving with respect to the m-chain constraints $u\left(n_{t}, D_{t}\right)_{l} \prec u\left(n_{t}, D_{t}\right)_{l^{\prime}}$ if $l<l^{\prime}$ for all $l=1, \ldots, D_{t}$ and $t=1, \ldots, m$. We call such a sequence by order-preserving sequence.

\section{LITERATURE SURVEY}

Steiner and Yeomans [20] solve the min-max problem reducing it to a single machine scheduling decision problem with release times and due dates. They represent it as a perfect matching in a $V_{\mathbf{1}}$-convex bipartite graph $G=\left(V_{\mathbf{1}} \mathbf{\cup} V_{2}, \mathbf{E}\right)$ where $\quad V_{\mathbf{1}}=\{1,2, \ldots, D\}$ denotes positions and $V_{2}=\left\{(i, j) \mid i=1,2, \ldots, n ; j=1,2, \ldots, d_{i}\right\}$ denotes the copies of the products. By this, if $\left(k^{{ }^{\prime}}, u\right),\left(k^{{ }^{\prime \prime}}, u\right) \in E$, then $(k, u) \in E$ with $k^{\dagger r}<k<k^{{ }^{\dagger \prime}}$. There exists an edge $\{k,(i, j)\} \in E$ if and only if $\mathrm{k}$ lies in the interval $[E(i, j), L(i, j)] \subseteq V_{\mathbf{1}}$ of release time and due date for the $j-$ th copy of the product $i$. Steiner and Yeomans [20] prove the following results (see also [2]).

Lemma 1 Let $d_{1}, \ldots, d_{n}$, be any instance of min-max-absolute problem. A sequence $s=s_{1} s_{2} \ldots s_{D}$ is $B$-feasible if and only if for all $i=1,2, \ldots, n$ and $j=1, \ldots d i$, this sequence assigns the copy $(i, j)$ to the interval $[E(i, j), L(i, j)]$, where $E(i, j)=\left(\left|\frac{j-B}{r_{i}}\right|\right)$ and $L(i, j)=\left|\frac{j-\mathbf{1}+B}{r_{i}}+\mathbf{1}\right|$ denote the release date and the due date of the copy $(i, j)$ for given upper bound $B$

Glover's [11] $\mathrm{O}(|\mathrm{E}|)$ modified Earliest Due Date algorithm is applied for finding a maxi-mum matching in the $\mathrm{V}_{\mathbf{1}}$-convex bipartite graph $G=\left(V_{1} \mathbf{U} V_{2}, \mathbf{E}\right)_{\text {such }}$ that each ascending $k \in V \mathbf{1}$ is matched to the unmatched copy $(i, j)$ with smallest due date $L(i, j)$.

The is tight lower bound for the min-max-absolute problem.

Theorem 1 An optimal solution can be determined by an exact pseudopolynomial algorithm with complexity $O(D \log D)$.

The sets of optimal sequences for the min-max-absolute and the min-max-squared problems include cyclic sequences, $[19,6]$, reducing the computational time.

There exist dynamic programming and heuristic algorithms for solving the mixedmodel just-in-time sequencing problems, see [7].

The derivation of similar closed formulas given by Lemma 1 for other measures of deviations was asked by [9]. Dhamala et al. [6] solved the open question of [9] 
and presented the closed formula (cf. Lemma 2) for the squared deviation objective function of the mixed-model just-in-time sequencing problem.

Lemma 2 Let $B$ be the given target value for the squared deviation objective function. Then, for $i=1, \ldots, n ; j=1, \ldots, d_{i}$, the unique integers $E(i, j)=\left\lceil\frac{j-\sqrt{B}}{r_{i}}\right\rceil$ and $L(i, j)=\left\lfloor\frac{j-1+\sqrt{B}}{r_{i}}+\mathbf{1}\right\rfloor$ define the feasible interval.

Any instance of the min-max problem has a feasible sequence if and only if, the $\mathrm{V} \mathbf{1}$ convex bipartite graph formed by the instance has an order-preserving perfect matching.

With these calculated bounds B and the necessary modifications on the solution method already applied for the bottleneck product rate variation problem with absolute deviation objective in [20,2], Dhamala et al. [6] solved the min-max problem with squared deviation objective function. The time complexity is pseudo-polynomial (cf. Theorem 2), which improves the previous solution approaches.

Theorem 2 A bisection search algorithm applied in the interval $\left[\left(1-r_{\max }\right)^{2},\left(1-\frac{1}{D}\right)^{2}\right]$ obtains an optimal sequence of the squared deviation sequencing problem with time complexity $O(D \log D)$.

The optimal $B^{*}$ satisfies the inequality $B^{*} \leq 1-\max \left\{\frac{1}{D}, \frac{1}{2(n-1)}\right\}$, for any demand $d_{i}, i=1,2, \ldots, n(n \geq 2)$ of the absolute objective. For $n \geq 3$, an instance with $\operatorname{gcd}\left(d_{1}, d_{2}, \ldots, d_{n}\right)=1$ has optimal $B^{*}=\frac{2^{n-1}-1}{2^{n}-1}<\frac{1}{2}$ if and only if $d_{i}=2^{i-1}$ for $i=1,2, \ldots, n,[2,3,16]$.

Dhamala [5] uses these methods to solve min-max problem with squared deviation and chain constraints. Khadka and Dhamala [13] define the following objective function

$$
f_{i}\left(x_{i k}-r_{i} k\right)=\| x_{i k}-r_{i} k^{m}
$$

for any positive integer $m$, denoted by $F_{m}$ and obtain the explicit formula for the required target value for this general objective. With this they show that the problem with this objective can be solved with the same time complexity as the other particular min-max objectives.

Some modifications on the lower and upper bounds are expressed to show the validity of the similar results for small deviations and the weighted objectives.

Our algorithm solves the min-max mixed-model just-in-time sequencing problem with this general objective and the chain constraints defined above. 


\section{AN EFFICIENT ALGORITHM}

We consider the demand rates of $n=\sum_{t=1}^{m} n_{t}$ models with total de mand $D=\sum_{t=\mathbf{1}}^{m} D_{t}$ of all chains. Then we calculate the permissible time windows $[E(i, j), L(i, j)]$ for given target value $B$, for our generalized objective (see, [13]). These time windows must be feasible without chains as these constraints were not included for calculating them yet.

To ensure that the target variable value $B$ for the bottleneck objective is feasible for the super sequence to be delivered, we reduce min-max-chain sequencing problem for objective $F_{m}$ to a single machine scheduling decision problem with release times, due dates and chain constraints.

Given any bound $B$ for the $F_{m}$ problem, we ask does there exists a feasible solution of the single processor scheduling problem 1|ri,chain|Lmax with $L \max \leq \mathbf{0}$ ?

For the problem $1 \mid r_{i}$, chain| $L_{\max }$, the time windows are represented by the intervals $\left[r_{i}, d_{i}\right]=[E(i, j), L(i, j)]$ calculated as a function of given $B$. The chains are given by the subsequences $\bigcup_{t=1}^{m}\left\{u\left(\left[n_{t}, D_{t}\right) \rrbracket_{l=1}^{D_{t}}\right\}\right.$ that may be represented by the following graph.

Define a directed graph $\mathcal{G}=(\mathcal{V}, \mathcal{E})$ with the vertex set $v=\bigcup_{t=1}^{m}\left\{u\left(\llbracket n_{t}, D_{t}\right) \rrbracket_{l=1}^{D_{t}}\right\}$. There exists an arc in $\varepsilon$ from $u\left(n_{t}, D_{t}\right)_{k}$ to $u\left(n_{t}, D_{t}\right)_{k^{\prime}}$ if the precedence relation $u\left(n_{t}, D_{t}\right)_{k} \prec u\left(n_{t}, D_{t}\right)_{k^{\prime}}$ is satisfied.

Horn [12] formulated $O(n \log n)$ time algorithm to the problem $\mathbf{1}\left|r_{i}, \operatorname{chain}\right| L_{\max }$. His earliest due date (EDD) rule assigns at any time an available job with the smallest due date.

For its use to $1 \mid r_{i}$, chain $L_{\max }$, one needs to modify the due dates as follows: if job $k$ is the immediate predecessor of job $l$ in any chain and $d_{k}^{l}=d_{l}-1<d_{k}$, denoted by $k \rightarrow l$, then the due date $d_{k}$ has to be replaced by the modified due date $d_{k}^{l}$.

We present Algorithm 1 for the $F_{m}$ problem (see also, $[5,8]$ ).

\section{Algorithm 1 min-max- $F_{m}$-chain-algorithm}




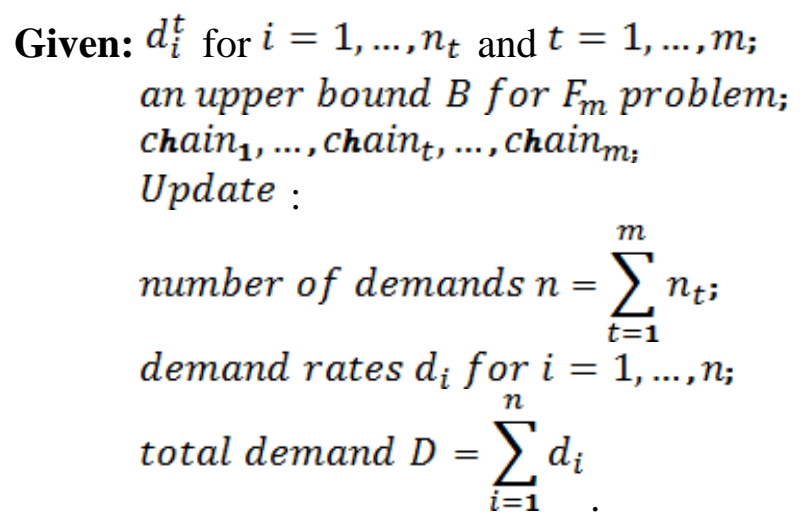

Step 1:

Calculate the windows $[E(i, j), L(i, j)]$ for $j=1, \ldots$, di and $i=1, \ldots, n$ by

Step 2: Modify the due dates $L(i, j)$ :

If $(i, j) \rightarrow\left(i^{\prime}, j^{\prime}\right)$, then

$L(i, j)=\min \left\{L(i, j), L\left(i^{\prime}, j^{\prime}\right)-\mathbf{1}\right\}$.

Step 3: Schedule the jobs by modified EDD lgorithm of [12].

Output: Target bound $B$ for $(n, D)$ if Lmax $\leq \mathbf{0}$.

The computational time complexity of the algorithm is $O(D \log D)$. Following theorem proves the correctness of Algorithm 1.

Theorem 3 Let $\mathrm{B}$ be a target value for the objective function of min-max-chain Fm problem. Then, if the modified EDD algorithm finds an optimal solution with $L_{\max } \leq 0$, then min-max-chain-algorithm finds a $\mathrm{B}$-feasible solution to min-maxchain $F_{m}$ problem.

Proof. Suppose that $s=s_{1} s_{2} \ldots s_{D}$ be a sequence obtained by min-max-chainalgorithm such that $\operatorname{Lmax} \leq 0$. That is, each job $k=1, \ldots, D$ is scheduled in the proper window and none of the job is delayed. If $s$ is infeasible to min-max-chain problem, then $f_{i}\left(x_{i k}-r_{i} k\right)>B$ for some product copy $(i, j)$ with $k=1, \ldots, D$ and $i=1, \ldots, n$. But this is impossible by the constriction of time windows.

An optimal solution to the min-max $-F_{m}$ problem has to be determined by applying binary search of the target value $B$ in the intervals determined by [13].

One way to give an upper bound to the obtained super sequence is to put given 
sequences $\bigcup_{t=1}^{m}\left\{u\left(\left[\left(n_{t}, D_{t}\right) \rrbracket_{l=1}^{D_{t}}\right\}\right.\right.$ one after another and then calculate the following value:

$B=\max _{i, k}\left\{f_{i}\left(x_{i k}-r_{i} k\right): \forall i=1,2, \ldots, n ; k=1,2, \ldots, D\right\}$.

Upper bounds calculated in this way are better, but the process is more implicit. How- ever, an explicit upper bound obtained, through the study of the properties of batch sequences, on the target value of the super sequence $s$ for the absolute deviation chain problem is $d_{\max }\left(1-r_{\max }\right)$ [8].

As $1-r_{\max }$ is the tight lower bound, he proves the following theorem. Similar results, of course with explicit upper bound, are applicable for the problem with objective $F_{m}$.

Theorem 4 An optimal solution to the min-max-absolute-chain problem can be deter- mined testing at most $O(D d \max (1-\operatorname{rmax}))$ sequences each with complexity $O(D \log D)$.

Proof. An optimal solution to the min-max-absolute-chain problem can be determined by applying the algorithm binary search in the interval $[1-r \max , d \max (1-r \max )]$. But a feasibility test requires $O(D \log D)$ time.

Horn's [12] algorithm works for the problem 1|ri,prec|Lmax. Therefore, our approach is applicable to the min-max $-F_{m}$ problem with precedence constraints as well. The time complexity of the algorithm does not increase.

\section{CONCLUSIONS}

A number of non-overlapping sequences in the mixed-model just-in-time production systems as chain constraints are added. We presented a pseudo-polynomial time algorithm which finds a minimum sequence to the maximum deviation mixedmodel just-in-time sequencing problem $F_{m}$.

These results are based on the reduction of mixed-model just-in-time sequencing problem to a single machine scheduling problem. Results of Horn [12] and Khadka and Dhamala 13] are applicable for solving our problem.

Our approach has both theoretical as well as practical values. The min-sum problem with such constraints and/or min-max problem with overlapping sequences as constraints will be interesting for further research.

\section{ACKNOWLEDGEMENTS}


The author would like to thank DAAD for the support of research visit at the University of Magdeburg, Germany (May-June, 2010).

\section{REFERENCES}

[1] Boysen N, Fliedner M \& Scholl A, Sequencing mixed-model assembly lines: Survey, classification and model critique. European Journal of Operations Research 192 (2009) 349.

[2] Brauner N \& Crama Y, The maximum deviation just-in-time scheduling problem. Discrete Applied Mathematics 134 (2004) 25.

[3] Brauner N, Jost V \& Kubiak W, On symmetric Fraenkel's and small deviations conjecture. Les cahiers du Laboratoire Leibniz-IMAG, 54, Grenoble, France, 2004.

[4] Corominas A \& Moreno N, On the relations between optimal solutions for different types of min-sum balanced JIT optimization problems. INFOR 41 (2003) 333-339.

[5] Dhamala TN, Optimality of the min-max product rate square deviation product rate variation problem with chain constraints, in Proceedings of the 14th International Workshop on Multimedia Signal Processing and Transactions, Chonbuk National University, South Korea, November 8 (2010) 94.

[6] Dhamala TN, Khadka SR \& Lee MH, A note on bottleneck product rate variation problem with square-deviation objective. International Journal of Operations Research 7 (2010) 1.

[7] Dhamala TN \& Khadka SR, A review on sequencing approaches for mixedmodel just-in-time production system. Iranian Journal of Optimization 1 (2009) 266.

[8] Dhamala TN, Just-in-time sequencing algorithms for mixed-model production systems. The Nepali Mathematical Sciences Report 24 (2005) 25.

[9] Dhamala TN \& Kubiak W, A brief survey of just-in-time sequencing for mixedmodel systems. International Journal of Operations Research (IJOR) 2 (2005) 1.

[10] Dhamala TN \& Kubiak W, Optimal just-in-time sequences for mixed-model multi-level production. Memorial University of Newfoundland (2005).

[11] Glover F, Maximum matching in a convex bipartite graph. Naval Research Logistics Quarterly, 4 (1967) 313.

[12] Horn WA, Some simple scheduling algorithms. Naval Research Logistics Quarterely 21 (1974) 177. 
[13] Khadka SR \& Dhamala TN, Optimality of the bottleneck product rate variation problem with a general objective. Operations Research Letters, under review (2010).

[14] Kotani S, Ito T \& Ohno K, Sequencing problem for a mixed-model assembly line in the Toyota production system. International Journal of Production Research, 42 (2004) 4955.

[15] Kovalyov MY, Kubiak W \& Yeomans JS, A computational study of balanced JIT optimization algorithms. Information Processing and Operational Research, 39 (2001) 299.

[16] Kubiak W, On small deviations conjecture. Bulletin of the Polish Academy of Sciences, 51 (2003) 189.

[17] Miltenburg J, Level schedules for mixed-model assembly lines in just-in-time production systems. Management Science, 35 (1989) 192.

[18] Monden Y, Toyota production systems. (Industrial Engineering and Management Press, Norcross, GA, 1983).

[19] Steiner G \& Yeomans S, Optimal level schedules in mixed-model multi- level JIT assembly systems with pegging. European Journal of Operational Research, 95 (1996) 38.

[20] Steiner G \& Yeomans S, Level schedules for just in time production processes. Management Science, 39 (1993) 728. 